\title{
The Survey of the Compliance Situation to the Antihypertensive Therapy Guideline by Analyzing Japanese National Claims Data
}

\author{
Hiromi Hagiwara, Ryouhei Nishikawa, Kazuki Fukuzawa, and Masahiro Tohkin* \\ Department of Regulatory Science, Graduate School of Pharmaceutical Sciences, \\ Nagoya City University; 3-1 Tanabe-dori, Mizuho-ku, Nagoya 467-8603, Japan.
}

(Received February 12, 2017; Accepted March 10, 2017)

\begin{abstract}
Kidney disease (KD) is a serious risk factor for cardiovascular event, and it is important to protect the heart and kidneys during treatment of the high blood pressure to prevent cardiovascular event. Japanese guideline (JSH2014) suggests using combination therapy to reduce the risk of comorbidities rather than high-dose monotherapy for the patients with cardiovascular disease and KD. Therefore, the present study assessed antihypertensive prescription patterns in Japanese patients with ischemic heart disease related diseases (IHDRD) and KD, and evaluated whether the prescription patterns match with the guideline-suggested therapies by analyzing the national insurance claims database (NDB). We extracted antihypertensive prescription patterns among Japanese IHDRD patients from the data of October 2011 of NDB, and examined the effect of KD on the prescription patterns. The number of prescribed antihypertensive was associated with KD among patients regardless of IHDRD. Patients with IHDRD and KD were more frequently prescribed combination therapy (calcium channel blockers/angiotensin II receptor blockers) than the calcium channel blocker monotherapy, based on the JSH2014. On the other hand, we did not observe the standard use of diuretics for patients with heart failure, which is suggested by the JSH2014. These findings suggested that patients with IHDRD and KD were frequently prescribed combination therapy to achieve its cardioprotective and renoprotective effects, according to the JSH2014, but the prescription profile to the patients with heart failure didn't match that of guideline-suggested therapies. This study provided a clinically important information and demonstrated the utility of NDB for compliance assessment for therapeutics guideline.
\end{abstract}

Key words_ antihypertensive prescription; claims database; guidelines; ischemic heart disease related diseases; kidney disease

\section{BACKGROUND}

The clinical guideline consists of evidence from clinical study. The survey of compliance with the guideline leads to assess appropriate therapy and the thinking of medical professionals to evidence-based medicine. Therefore, we survey the compliance of guideline using large scale medical database in order to promote appropriate medication therapy.

The heart and kidneys are functionally linked by the sympathetic nervous system, renin-angiotensin-aldosterone system, and antidiuretic hormone. Because the kidneys regulate blood pressure (BP) through hormonal control of water volume and ion concentrations, kidney disease (KD) and hypertension create a vicious cycle. Therefore, $\mathrm{KD}$ is an important risk factor in the progression of cardiovascular events, as well as the most common comorbidity in cases of cardiovascular disease (CVD). Increased risk of

\footnotetext{
*e-mail: tohkin@phar.nagoya-cu.ac.jp
}

CVD induced the hypertension which exacerbate the serious KD. Therefore, CVD is also a risk factor in the progression of serious KD. CVD includes ischemic heart disease related diseases (IHDRD). In this study, we defined KD as risk factor of IHDRD, and we examined the effect of KD on the prescription patterns of IHDRD patients. These mechanisms highlight the importance of protecting the heart and kidneys during treatment to control $\mathrm{BP}$, and the BP of patients with CVD and KD should be controlled more strictly than that of patients with only CVD. ${ }^{1,2)}$ In addition, antihypertensive therapy is thought to provide protection against stroke, heart failure, and renal disease. In fact, the Japanese guideline for antihypertension management (JSH2014) suggests combination therapy, rather than monotherapy, to achieve optimal BP control in patients with CVD and KD. ${ }^{2-8)}$

In Japan, $99.3 \%$ of Japanese residents are covered by the national health insurance system, ${ }^{9)}$ and this system requires that medical institutions provide 


\begin{tabular}{|c|c|c|c|c|}
\hline \multicolumn{5}{|c|}{ Medical claims } \\
\hline RE record & $\begin{array}{l}\text { Reference Number } \\
(\mathrm{RN})\end{array}$ & sex & age & ID-1 \\
\hline IY record & $\mathrm{RN}$ & $\begin{array}{l}\text { Medication } \\
\text { name }\end{array}$ & & \\
\hline SY record & RN & diagnoses & modifiers & 1 \\
\hline \multicolumn{5}{|c|}{ Dispensation claims } \\
\hline $\mathrm{RE}$ record & $\begin{array}{l}\text { Reference } \\
\text { Number (RN) }\end{array}$ & sex & age & ID-1 \\
\hline IY record & RN & $\begin{array}{l}\text { Medication } \\
\text { name }\end{array}$ & & \\
\hline
\end{tabular}

Fig. 1. The Structure and Restructuring Strategies of Claims Data

The structure of the medical and dispensed claims, and the restructuring strategies based on the patients' records. The medical and dispensed claims consisted of records that were stored in the claims database. We reconstructed these claims using the patient's reference number, and then combined the medical and dispensed claims by matching their common identification number-1 (ID-1) for outpatients whose medication was dispensed in a pharmacy. RE record: ID-1, age group, sex, month, and reference number; SY record: diagnoses, their modifiers, and reference number; IY record: medication name and reference number.

itemized claims for each patient. The Japanese Ministry of Health, Labour and Welfare (MHLW) collects the claims data and has created an electronic National Insurance Claims and Health Checkup Database (NDB) . ${ }^{10)}$ Because the NDB includes the largest collection of Japanese medical data, regardless of the patients' region or income, this database is suitable for surveys of Japanese pharmacoepidemiology. ${ }^{11,12}$ The present study attempted to clarify the antihypertensive prescription patterns for patients with IHDRD and KD, and to compare these patterns to the guideline-suggested therapy for antihypertensive treatment among patients with IHDRD and KD.

\section{METHODS}

Study Database The MHLW provided a NDB sampling data set from October 2011, which included $10 \%$ of all inpatient medical claims and $1 \%$ of all outpatient medical claims. The study period (October 2011) was arbitrarily selected by the MHLW, and we were unable to request a more recent dataset. However, the data remain relevant, as they highlight the approach of healthcare professionals to understanding and implementing national guideline. Although the period (2011) of NDB data used in this study does not match with the issue date of JSH2014, this different timing does not affect our study purpose because the guideline-2014 is not different from the guideline-2009 regarding discussion point of this study, i.e., the policy to KD patients.

The present study's design was reviewed and approved by the ethics committee of Nagoya City
University and the National Database Committee of the MHLW. The NDB contains medical, dispensation, and dental claims. Each claim is divided into several records, which are then uploaded to the NDB. The MHLW had anonymized all data by removing identifiable information regarding the included patients and medical institutions, and we used a common anonymous identification number (ID-1) to establish links between the claims. In addition, all records from one claim have the same reference number (RN).

Medical claims from clinics and hospitals include $\mathrm{RE}$ records with general demographic information (ID-1, age group, sex, month, and RN), SY records with disease information (diagnoses and their modifiers, and RN), and IY records with medication information (medication name and RN). The dispensation claims from pharmacies contain outpatient RE records (ID-1, age group, sex, month, and RN) and IY records (medication name and $\mathrm{RN}$ ). Figure 1 shows the structure of the dispensation and medical claims data, as well as how we combined the dispensation claim records with the outpatients' medical claims.

Data Cleaning and Analysis Because the extraction rates for the inpatient and outpatient claims data were different, and because outpatients have a unique dispensation route ( $v s$. inpatients), we separated the inpatient and outpatient data. To analyze each patient's claims data from the NDB data set, we reconstructed all relevant medical claims using the patient's RN. However, we omitted medical claims 


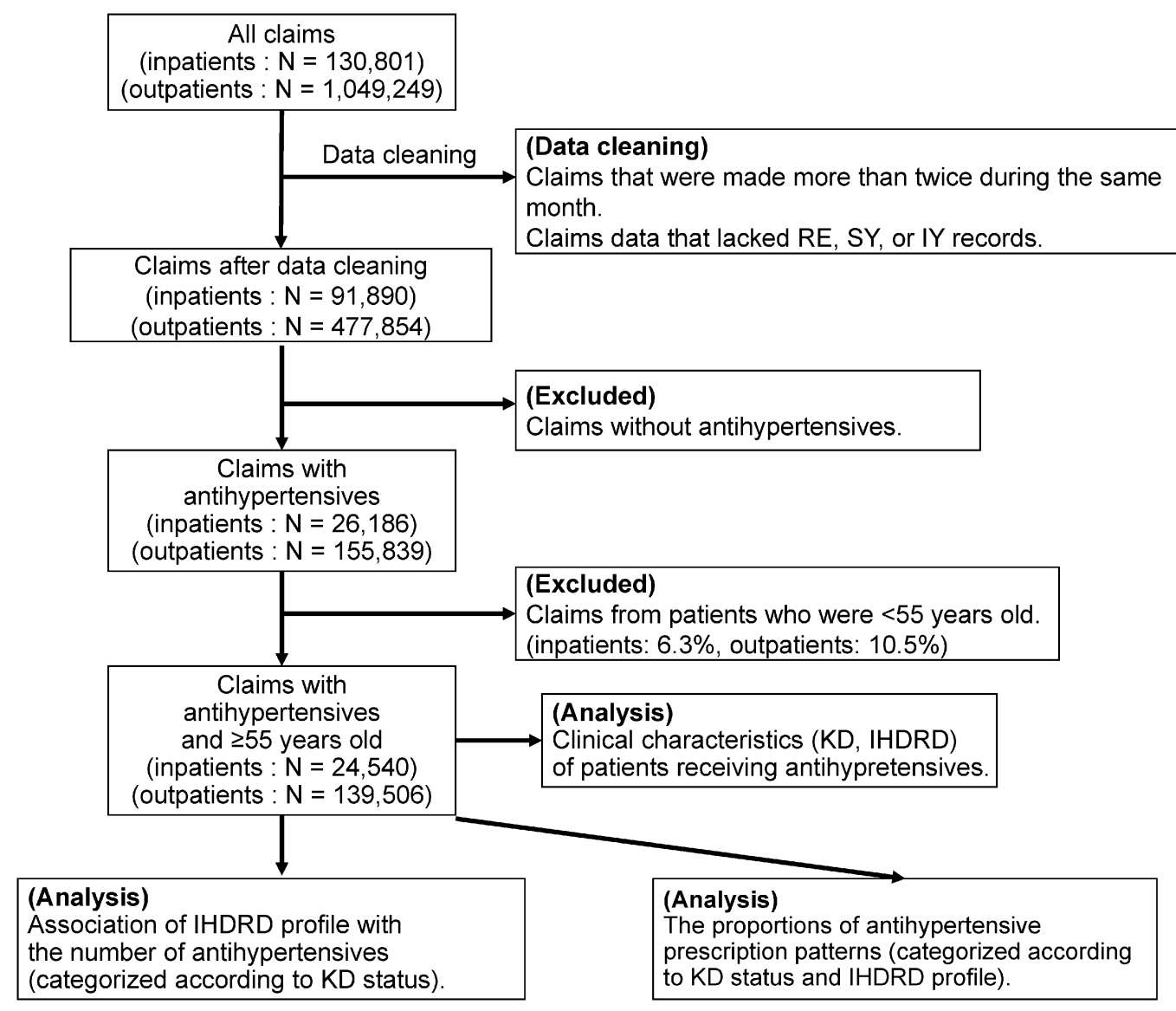

Fig. 2. The Patient Selection Flowchart

RE record: ID-1, age group, sex, month, and reference number; SY record: diagnoses, their modifiers, and reference number; IY record: medication name and reference number; IHDRD: ischemic heart disease related diseases; KD: kidney disease.

data that did not include an ID-1 identifier and/or all information for the RE, SY, and IY records. We also linked outpatient medical claims from the hospitals with the pharmacy dispensation claims, although we omitted outpatient medical claims data that occurred more than twice in the same month, because we could not determine which medical claim corresponded to the dispensation claim. We also omitted outpatient dispensation claims data that had the same ID-1 that was listed on the omitted medical claims. The outpatient dispensation claims data were reconstructed by combining the RE and IY records using the RN, and we reconstructed the outpatient medical claims by combining the RE, SY, and IY records using the $\mathrm{RN}$. Thus, after excluding cases without an ID-1 identifier, the ID-1 could be used to match the medical and dispensation claims for the included patients.

After the data cleaning was performed, we choose antihypertensive agents in IY records (medication name). The antihypertensive treatments were divided into calcium channel blockers (CCB), angiotensin II receptor blockers (ARB), angiotensin converting enzyme inhibitors (ACEI), $\beta$-blockers (BB), loop diuretics, other diuretics, thiazide, carbonate dehydratase inhibitors, and other antihypertensives. For example, the compounding agent which consists of $\mathrm{CCB}$ and ARB was counted as 2 types of antihypertensives. We subsequently removed data from patients who were $<55$ years old $(<10 \%$ of the sample population) to exclude cases of juvenile hypertension. We extracted IHDRD and/or KD from SY records (diagnoses). We defined cases of IHDRD as cases that involved hyperlipidemia, arteriosclerosis, angina pectoris, and/or heart failure. If the patient has more than 2 records (e.g. hyperlipidemia and heart failure), we counted as more serious disease (e.g. heart failure) patient. KD was defined using codes that contained the words "kidney disease" and kidney disease's words from the 10th revision of the International Statistical Classification of Diseases and Related Health Problems, which were associated with a reduction in the estimated glomerular filtration rate. 
Table 1. Demographic Data from the National Health Insurance Claims Database

\begin{tabular}{|c|c|c|c|}
\hline \multirow[b]{2}{*}{ Variable } & \multicolumn{3}{|c|}{ No. (\%) } \\
\hline & $\begin{array}{c}\text { Before } \\
\text { data cleaning }\end{array}$ & $\begin{array}{l}\text { After } \\
\text { data cleaning }\end{array}$ & $\begin{array}{l}\text { Patients who } \\
\text { were prescribed } \\
\text { antihypertensives }\end{array}$ \\
\hline \multicolumn{4}{|l|}{ Inpatients $^{\mathrm{a}}$} \\
\hline Overall & $130801(100.0)$ & $91890(100.0)$ & $26186(100.0)$ \\
\hline \multicolumn{4}{|l|}{ Age (years) } \\
\hline $0-54$ & $28527(21.8)$ & $22720(24.7)$ & $1646(\quad 6.3)$ \\
\hline$\geq 55$ & $102274(78.2)$ & $69170(75.3)$ & $24540(93.7)$ \\
\hline \multicolumn{4}{|l|}{ Sex } \\
\hline Male & $56771(43.4)$ & $40561(44.1)$ & $11165(42.6)$ \\
\hline Female & $74030(56.6)$ & $51329(55.9)$ & $15021(57.4)$ \\
\hline \multicolumn{4}{|c|}{ Outpatients $^{\mathrm{b}}$} \\
\hline Overall & $1049249(100.0)$ & $477854(100.0)$ & $155839(100.0)$ \\
\hline \multicolumn{4}{|l|}{ Age (years) } \\
\hline $0-54$ & $409154(39.0)$ & $212755(44.5)$ & $16333(10.5)$ \\
\hline$\geq 55$ & $640095(61.0)$ & $265099(55.5)$ & $139506(89.5)$ \\
\hline \multicolumn{4}{|l|}{ Sex } \\
\hline Male & $443390(42.3)$ & $212107(44.4)$ & $72929(46.8)$ \\
\hline Female & $605859(57.7)$ & $265747(55.6)$ & $82910(53.2)$ \\
\hline
\end{tabular}

a Inpatients' data were extracted from $10 \%$ of all inpatient medical

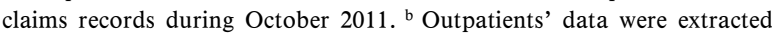
from $1 \%$ of all outpatient medical claims records during October 2011.

The order of the extractions steps is shown in Fig. 2 . All analyses were performed using SAS software (version 9.4; SAS Institute Inc., Cary, USA). Odds ratios (OR) and 95\% confidence interval (CI) are reported for significant associations. All significant tests were Chi-square test, and statistical significance was assumed as $p<0.05$.

\section{RESULTS}

\section{Clinical Characteristics of Patients Who Received} Antihypertension Therapy We evaluated data from 91890 inpatient claims and 477854 outpatient claims (Table 1). The data cleaning procedures excluded $29.7 \%$ of the inpatient medical claims, $54.5 \%$ of the outpatient medical claims, and $35.7 \%$ of the dispensation claims. We can use about $50 \%$ of all outpatients' claims data because medical claims and dispensation claims could not be combined. Thus, we had available data of 26186 inpatients (28.5\%) and 155839 outpatients $(32.6 \%)$ who received antihypertension therapy. This data indicated that the men were more likely to have hypertension, although we did not perform sex-specific analyses because previous studies have demonstrated that there are no sex- specific differences in the ability of antihypertensive therapy to reduce the risk of cardiovascular event. ${ }^{13-15)}$ We categorized the included patients according to their progression of IHDRD diagnoses (hyperlipidemia, arteriosclerosis, angina pectoris, heart failure, and non-IHDRD), and evaluated the proportions of KD according to IHDRD classification (Table 2). Compared to outpatients, inpatients exhibited higher proportions of the different IHDRD and KD comorbidities. The comorbidities' proportion of heart failure and KD (inpatients: $36.0 \%$, outpatients: 21.6\%) was highest than other patients' groups.

Comparing Combination Therapy with Monotherapy among Patients with IHDRD and KD The JSH2014 suggests that BP control for patients with IHDRD and KD should involve combination therapy to protect the kidneys and heart. Therefore, we examined the association of $\mathrm{KD}$ with the number of prescribed antihypertensives among patients with or without IHDRD (Fig. 3). Monotherapy was more common among patients with only IHDRD, and both inpatients and outpatients with IHDRD and KD exhibited higher proportions of combination therapy (vs. patients with IHDRD alone) (Fig. 3 Left panel) . Monotherapy was more common among patients without IHDRD, too, and both inpatients and outpatients with only KD exhibited higher proportions of combination therapy ( $v s$. patients without KD) (Fig. 3 Right panel). Ridit analyses revealed that the number of prescribed antihypertensive was significantly higher among patients with $\mathrm{KD}$, compared to patients without KD ( $p<0.05$ after Bonferroni correction).

Associations of KD with CCB Monotherapy and CCB/ARB Combination Therapy We examined the most frequently prescribed antihypertensives by analyzing $\mathrm{NDB}$, and $\mathrm{CCB}$ was most frequently prescribed antihypertensive class. And also about 2 combination therapy, CCB/ARB therapy was most frequently prescribed therapy (data not shown). Therefore, we focused on CCB and ARB more than other antihypertensives. Previous studies have suggested that the addition of ARB to CCB therapy was more effective than CCB monotherapy for preventing cardiovascular events and KD progression. ${ }^{16-19)}$ Therefore, we examined the associations of KD with CCB monotherapy and combination therapy including CCB and ARB (Table 3). Significant associations with KD status and the treatment groups were ob- 
Table 2. The Number of Patients with Antihypertensive Prescriptions According to Ischemic Heart Disease Related Diseases and Kidney Disease Status

\begin{tabular}{lcccccc}
\hline \hline & Overall & Hyperlipidemia & Arteriosclerosis & Angina pectoris & Heart failure & Non-IHDRD \\
\hline Inpatients $n$ & 24540 & 2639 & 816 & 3411 & 9796 & 7878 \\
With KD $n(\%)$ & $6491(26.5)$ & $490(18.6)$ & $272(33.3)$ & $875(25.7)$ & $3527(36.0)$ & $1327(16.8)$ \\
\hline Outpatients $n$ & 139506 & 41900 & 6036 & 16235 & 21630 & 53705 \\
With KD $n(\%)$ & $16535(11.9)$ & $4717(11.3)$ & $1074(17.8)$ & $2459(15.1)$ & $4666(21.6)$ & $3619(6.7)$ \\
\hline
\end{tabular}

IHDRD, ischemic heart disease related diseases; KD, kidney disease.
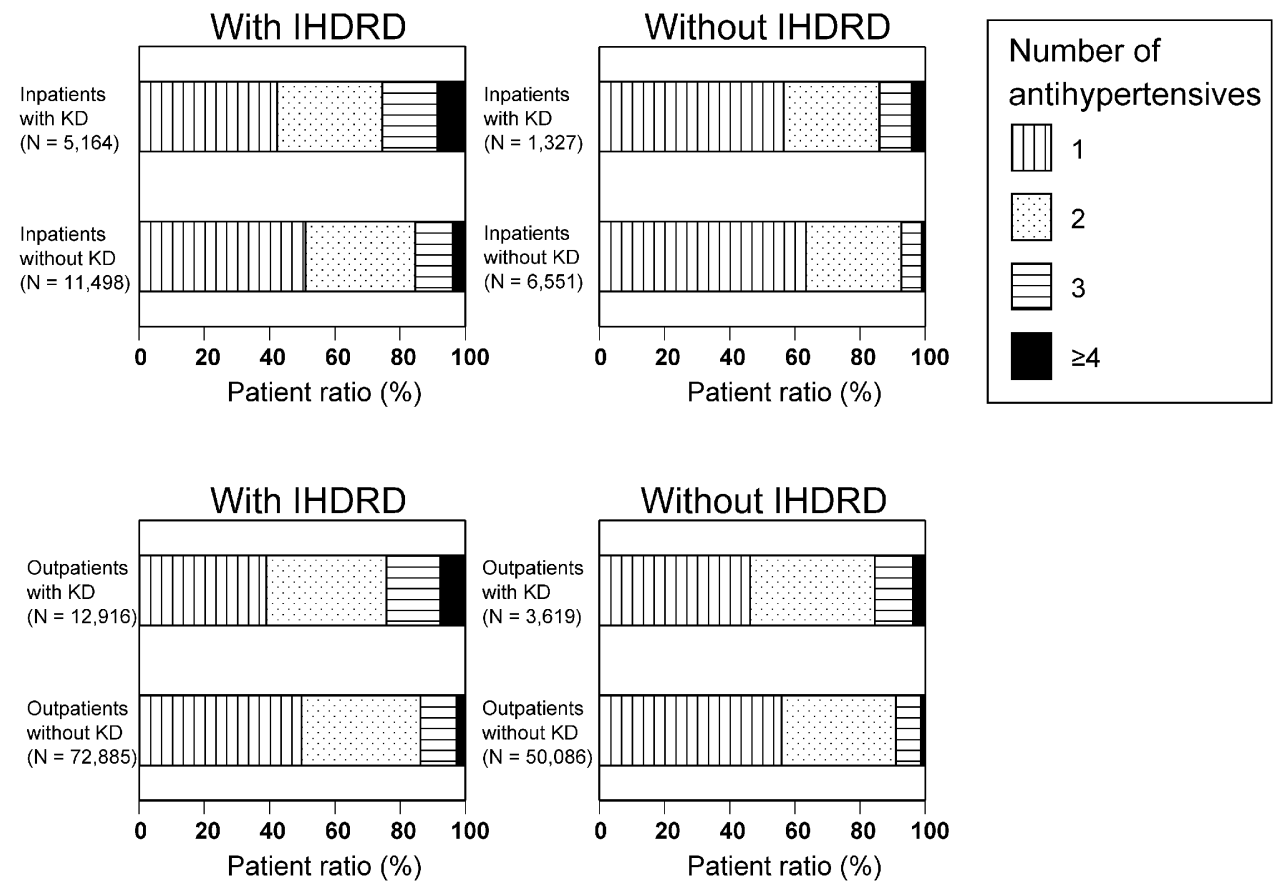

Fig. 3. The Patient's Ratio of Antihypertensive Therapies

The ratios of monotherapy and combination therapies among patients with ischemic heart disease related diseases (IHDRD) and according to their kidney disease (KD) status. Ridit analyses were performed to compare the effects of KD status on the number of antihypertensives prescribed to patients with IHDRD. $p<$ 0.001 was considered statistically significant after Bonferroni correction for multiple comparisons.

served among outpatients with all forms of IHDRD diagnoses. These associations were also observed among inpatients with hyperlipidemia, angina pectoris and heart failure. These results suggested that there was a significant association between the presence of KD (regardless of comorbid diseases) and $\mathrm{CCB} / \mathrm{ARB}$ therapy, which indicates that patients with $\mathrm{KD}$ were typically prescribed $\mathrm{CCB} / \mathrm{ARB}$ therapy, rather than CCB monotherapy.

Prescribing Patterns among Patients with Heart Failure It is very important to select appropriate medication for body fluid regulation in patients with heart failure. Therefore, we investigated the variations of antihypertensive drug classes in the heart failure subgroup (Table 4). The JSH2014 suggests that combination therapy [a rennin-angiotensin inhib- itor (RA inhibitor) with a $\mathrm{BB}$ and a diuretic] be used as the standard therapy to improve prognosis in patients with heart failure. However, results of Table 4 indicate that $<0.3 \%$ of the patients with heart failure received this combination, even among patients with KD. Moreover, inpatients with heart failure were most likely to be prescribed loop diuretic monotherapy and outpatients with heart failure were most likely to be prescribed ARB/CCB therapy. To examine whether heart failure was associated with the selection of combination therapy using diuretics, we compared the ARB/diuretics therapy (using loop diuretics, thiazide, and other diuretics), which is suggested by the JSH2014, and CCB/ARB therapy, which was the most commonly prescribed combination therapy for patients with heart failure (Table 5). 
We did not observe any significant associations between KD status and the antihypertensive therapies (ARB/diuretics therapy or ARB/CCB therapy) in inpatients (OR, 0.87; 95\% CI, 0.69-1.10) and outpatients (OR, 1.11;95\% CI, 0.95-1.29) with heart failure. However, KD status was significantly associated with the therapy modes (ARB/diuretics therapy and $\mathrm{ARB} / \mathrm{CCB}$ therapy) among inpatients and outpatients without heart failure. These results suggest that KD status was not significantly associated with the selection of diuretics-containing combination therapy among patients with heart failure.

\section{DISCUSSION}

Patients with IHDRD and KD have an elevated risk of cardiovascular events, and these patients should be controlled hypertension more strictly and considered to be prescribed combination therapy to minimize their risks of comorbidities. We hypothesized that prescription pattern should depend on the progression status of IHDRD. Since we cannot determine the diseases progression status from NDB, we assumed that the hyperlipidemia, arteriosclerosis and angina pectoris would be less severe phenotype than heart failure, and then evaluated the combination therapy ratios in the patients with heart failure comparing with three other diseases as a reference. We studied the patients with IHDRD and/or KD, who were treated mainly by CCB or ARB. Patients with myocardial infarction are treated mainly by $\mathrm{BB}$ and thrombolytic drug in most cases. Arrhythmia are not classified as IHDRD, and patients with arrhythmia are treated by other medication rather than CCB and ARB. Therefore, we excluded these patients with myocardial infarction and arrhythmia from this study. We evaluated the proportions of various comorbidities according to IHDRD class and KD status, which revealed that arteriosclerosis or heart failure with KD were the two most common comorbidities (Table 2). Next, we evaluated the prescription of monotherapy or combination therapy according to IHDRD and KD status, and observed that patients with IHDRD and KD were more likely to receive combination therapy, compared to patients with only IHDRD (Fig. 3). This pattern may reflect the guideline-suggested therapies, which indicate that serious patients need combination therapy in the most cases, and combination therapy is more effective for reducing the risk of comorbidities, compared to high-dose monotherapy. Therefore, it appears that KD was associated with combination therapy, which led us to examine the detailed antihypertensive prescription patterns according to comorbidity and based on the JSH2014.

Among patients with KD, BP control therapy aims to prevent cardiovascular events and inhibit KD progression. Thus, the JSH2014 suggests prescribing combination therapy for patients with KD, and to include ARB therapy for its ability to protect the heart, kidneys, and brain. This is because a previous study has suggested that the combination of $\mathrm{ARB} / \mathrm{CCB}$ therapy is more effective than CCB monotherapy for preventing IHDRD and KD progression. ${ }^{16-19)}$ Because preliminary examinations and comparisons by analyzing $\mathrm{NDB}$ showed that $\mathrm{CCB}$ and $\mathrm{CCB} / \mathrm{ARB}$ combination therapy (about 2 combination therapy) were most frequently prescribed therapy (data not shown), we focused on CCB and ARB more than other antihypertensives at this study. Then, we found a significant association between the presence of KD (regardless of comorbid diseases) and $\mathrm{CCB} / \mathrm{ARB}$ therapy, as patients with $\mathrm{KD}$ were more likely to receive $\mathrm{CCB} / \mathrm{ARB}$ therapy, compared to $\mathrm{CCB}$ monotherapy (Table 3 ). This result suggests that Japanese patients with KD are most frequently prescribed combination therapy, which reflects the JSH2014 evidence-based therapy to include ARB. However, we did not observe a significant association between the presence of comorbidities and $\mathrm{CCB}$ / ARB therapy among inpatients with arteriosclerosis, which might be due to a small number of patients and thus insufficient statistical power. In order to assess a possibility that a higher ratio of $\mathrm{CCB} / \mathrm{ARB}$ combination therapy might be due to a preferential prescription of ARB itself in the patients with KD, we examined an association of ARB monotherapy with KD status comparing with CCB monotherapy (data not shown). The result indicated no preferential association of ARB monotherapy in the patient with serious disease status, i.e., inpatients or patients with heart failure, which was in accordance with the current finding that serious patients were more frequently treated with CCB/ARB combination therapy.

Among patients with heart failure, it is difficult to balance the regulation of their fluid levels with the medication's effects on the patient's heart, kidneys, and prognosis. Furthermore, this balance is even more difficult to achieve among patients with KD, and the appropriate selection of antihypertensive therapy 
Table 3. Effects of Kidney Disease on CCB Monotherapy and Combination Therapy (Including CCB/ARB)

\begin{tabular}{|c|c|c|c|c|}
\hline \multicolumn{5}{|l|}{ INPATIENTS } \\
\hline \multirow[b]{2}{*}{ Drug class } & \multicolumn{2}{|r|}{ No. } & \multirow{2}{*}{$\begin{array}{c}\text { Odds Ratio } \\
(95 \% \mathrm{CI})\end{array}$} & \multirow[b]{2}{*}{$p$} \\
\hline & $\begin{array}{l}\text { With } \\
\text { KD }\end{array}$ & $\begin{array}{c}\text { Without } \\
\text { KD }\end{array}$ & & \\
\hline \multicolumn{5}{|l|}{ Non-IHDRD } \\
\hline CCB monotherapy & 296 & 1915 & \multirow{2}{*}{$\begin{array}{c}1.46 \\
(1.23-1.74)\end{array}$} & \multirow{2}{*}{$<0.05$} \\
\hline CCB/ARB $(\geq 2$ drugs $)$ & 313 & 1384 & & \\
\hline \multicolumn{5}{|l|}{ Hyperlipidemia } \\
\hline CCB monotherapy & 92 & 565 & \multirow{2}{*}{$\begin{array}{c}1.69 \\
(1.28-2.23)\end{array}$} & \multirow{2}{*}{$<0.05$} \\
\hline CCB/ARB $(\geq 2$ drugs $)$ & 173 & 630 & & \\
\hline \multicolumn{5}{|l|}{ Arteriosclerosis } \\
\hline CCB monotherapy & 49 & 114 & \multirow{2}{*}{$\begin{array}{c}1.50 \\
(0.99-2.28)\end{array}$} & \multirow{2}{*}{0.054} \\
\hline CCB/ARB ( $\geq 2$ drugs $)$ & 104 & 161 & & \\
\hline
\end{tabular}

Angina pectoris

\begin{tabular}{lllc}
\hline CCB monotherapy & 167 & 697 & 1.67 \\
CCB/ARB $(\geq 2$ drugs $)$ & 267 & 666 & $(1.34-2.09)$
\end{tabular}$<0.05$

Heart failure

\begin{tabular}{lrrcr}
\hline CCB monotherapy & 358 & 739 & 1.70 \\
CCB/ARB $(\geq 2$ drugs $)$ & 850 & 1035 & $(1.45-1.98)$ & $<0.05$ \\
\hline
\end{tabular}

OUTPATIENTS

\begin{tabular}{lll}
\hline \multirow{3}{*}{ Drug class } & \multicolumn{2}{c}{ No. } \\
\cline { 2 - 3 } & $\begin{array}{c}\text { With Without } \\
\text { KD }\end{array}$ & $\begin{array}{c}\text { Odds Ratio } \\
\text { KD }\end{array}$ \\
\hline
\end{tabular}

\begin{tabular}{lrrcc}
\hline Non-IHDRD & & & & \\
\hline CCB monotherapy & 753 & 15859 & 1.78 & $<0.05$ \\
CCB/ARB $(\geq 2$ drugs $)$ & 1333 & 15747 & $(1.63-1.95)$ & \\
\hline
\end{tabular}

\begin{tabular}{lrrrr}
\hline Hyperlipidemia & & & & \\
\hline CCB monotherapy & 956 & 11253 & 1.82 & $<0.05$ \\
CCB/ARB $(\geq 2$ drugs $)$ & 1886 & 12227 & $(1.67-1.97)$ & \\
\hline Arteriosclerosis & & & & \\
\hline CCB monotherapy & 186 & 1206 & 1.64 & $<0.05$ \\
CCB/ARB $(\geq 2$ drugs $)$ & 446 & 1763 & $(1.36-1.98)$ & \\
\hline
\end{tabular}

\begin{tabular}{lrrcc}
\hline Angina pectoris & & & & \\
\hline CCB monotherapy & 446 & 4085 & 2.04 \\
CCB/ARB $(\geq 2$ drugs $)$ & 1037 & 4662 & $(1.81-2.29)$ & $<0.05$ \\
\hline
\end{tabular}

Heart failure

\begin{tabular}{lrrcr}
\hline CCB monotherapy & 492 & 2331 & 1.61 \\
CCB/ARB $(\geq 2$ drugs $)$ & 1702 & 5001 & $(1.44-1.80)$ & $<0.05$ \\
\hline
\end{tabular}

ARB, angiotensin II receptor blockers; $\mathrm{CCB}$, calcium channel blocker; CI, confidence interval; IHDRD, ischemic heart disease related diseases; $\mathrm{KD}$, kidney disease. is important in this subgroup. In the present study, many inpatients and outpatients with heart failure also had comorbid KD (Table 2), and these patients are considered difficult to treat. The JSH2014 suggests using combination therapy (an RA inhibitor with a BB and a diuretic) as the standard therapy for improving prognosis among patients with heart failure. Therefore, we examined the antihypertensive prescription patterns among the heart failure subgroup, which revealed that the rate of the standard combination therapy is very low (Table 4). Interestingly, a meta-analysis of hypertensive treatments revealed that diuretics and RA inhibitors were the most effective drugs for preventing heart failure, although CCBs and BBs were less effective. ${ }^{20)}$ Thus, the authors of these papers suggested that the first-line therapy for heart failure include the diuretic alone or the combination with a RA-inhibitor. Moreover, the previous study revealed that ARB inhibited the progression of KD. ${ }^{21)}$ JSH2014 suggests that patients with KD use diuretics in preference to CCB as RA inhibitor combination therapy in case that their fluid level is high. Therefore, we compared combination therapies using $\mathrm{ARB} /$ diuretics or $\mathrm{CCB} / \mathrm{ARB}$ (most frequent therapy) for patients with heart failure, which revealed that there was no significant difference in the two prescription rates (Table 5). Those results of Table 4 and 5 also supported that the patients with heart failure were not prescribed more preferentially the combination therapy including diuretics than other combination therapy. We could not confirm the actual therapy which match the guideline-suggested therapy by the analysis of NDB. Because the study using NDB cannot address the reason of the mismatch, we expect the further clinical survey including detail clinical symptom.

We consider that the post-marketing study using NDB is useful not only for the pharmacovigilance study, but also for the verification of therapeutics guideline. Because there are few researches using NDB, this study leads to further studies using NDB and large electronic medical database.

The present study has several limitations that warrant consideration. First, we cannot analyze the prescription patterns according to the progression or stage of clinical symptoms because the NDB does not include more detailed clinical findings. Second, the claims data provide only diagnostic codes, and we cannot determine the order or intensities of different 
Table 4. Effects of Kidney Disease on Prescribing Patterns among Patients with Heart Failure

\begin{tabular}{|c|c|c|c|}
\hline \multicolumn{4}{|c|}{ Inpatients with heart failure } \\
\hline \multirow{2}{*}{ Rank } & \multirow{2}{*}{ Drug class } & \multicolumn{2}{|c|}{ No. (\%) } \\
\hline & & With KD & Without KD \\
\hline 1 & Loop monotherapy & $713(20.2)$ & $1455(23.2)$ \\
\hline 2 & CCB monotherapy & $358(10.2)$ & $739(11.8)$ \\
\hline 3 & $\mathrm{ARB} / \mathrm{CCB}$ & $285(8.1)$ & $466(7.4)$ \\
\hline 35 & ARB/Loop/BB & $10(0.3)$ & $15(0.2)$ \\
\hline 49 & $\mathrm{ACEI} / \mathrm{Loop} / \mathrm{BB}$ & $<10(\quad 0.1)$ & $<10(0.1)$ \\
\hline 58 & $\mathrm{ARB} /$ thiazide/BB & $<10(\quad 0.1)$ & $<10(\quad 0.0)$ \\
\hline - & $\mathrm{ARB} / \mathrm{BB} /$ other diuretics & $<10(\quad 0.0)$ & $<10(\quad 0.0)$ \\
\hline - & Overall & $3527(100.0)$ & $6269(100.0)$ \\
\hline \multicolumn{4}{|c|}{ Outpatients with heart failure } \\
\hline \multirow{2}{*}{ Rank } & \multirow{2}{*}{ Drug class } & \multicolumn{2}{|c|}{ No. $(\%)$} \\
\hline & & With KD & Without KD \\
\hline 1 & $\mathrm{ARB} / \mathrm{CCB}$ & $654(14.0)$ & $2527(14.9)$ \\
\hline 2 & CCB monotherapy & $492(10.5)$ & $2331(13.7)$ \\
\hline 3 & Loop monotherapy & $406(8.7)$ & $1403(8.3)$ \\
\hline 25 & ARB/Loop/BB & $25(0.5)$ & $98(0.6)$ \\
\hline 43 & ACEI/Loop/BB & $<10(\quad 0.2)$ & $33(0.2)$ \\
\hline 46 & $\mathrm{ARB} /$ thiazide/BB & $<10(\quad 0.2)$ & $27(\quad 0.2)$ \\
\hline - & ACEI/thiazide/BB & $<10(\quad 0.0)$ & $<10(\quad 0.0)$ \\
\hline - & Overall & $4666(100.0)$ & $16964(100.0)$ \\
\hline
\end{tabular}

ACEI, angiotensin converting enzyme inhibitors; ARB, angiotensin II receptor blockers; $\mathrm{BB}, \beta$-blocker; $\mathrm{CCB}$, calcium channel blocker; $\mathrm{KD}$, kidney disease; Loop, loop diuretics. Rank: the ranking within the list of most commonly prescribed therapies.

therapies. Nevertheless, the NDB provides nationally representative data that are not biased by specific institutions, and it appears that these data are suitable for post-marketing surveillance. Third, the healthcare system in Japan has two claims systems: the regular system, which includes NDB, and the diagnosis procedure combination system (DPC system) for acute medical care. Although we did not analyze the DPC system data, which likely included some patients with IHDRD and KD, DPC may provide the useful data to analyze prescription pattern. Fourth, we cannot extract information of smoking, family history, and obesity from NDB because there are no information in the claims data. Therefore, these confounding factors could not be adjusted in this study. We also cannot adjust the age and the diabetes because these information are complicated and not accurate.
Table 5. Relationships between Kidney Disease and Combination Therapy (Including Diuretics) among Patients with Heart Failure

\section{INPATIENTS}

\begin{tabular}{|c|c|c|c|c|}
\hline \multirow{2}{*}{ Drug class } & \multicolumn{2}{|c|}{ No. } & \multirow{2}{*}{$\begin{array}{l}\text { Odds Ratio } \\
(95 \% \mathrm{CI})\end{array}$} & \multirow[b]{2}{*}{$p$} \\
\hline & $\begin{array}{l}\text { With } \\
\text { KD }\end{array}$ & $\begin{array}{l}\text { Without } \\
\text { KD }\end{array}$ & & \\
\hline \multicolumn{5}{|l|}{ Non-IHDRD } \\
\hline $\mathrm{ARB} /$ diuretics (2 drugs) & 35 & 125 & \multirow{2}{*}{$\begin{array}{c}1.66 \\
(1.11-2.50)\end{array}$} & \multirow{2}{*}{$<0.05$} \\
\hline $\mathrm{ARB} / \mathrm{CCB}(2$ drugs $)$ & 178 & 1056 & & \\
\hline \multicolumn{5}{|l|}{ Heart failure } \\
\hline $\mathrm{ARB} /$ diuretics (2 drugs) & 169 & 318 & \multirow{2}{*}{$\begin{array}{c}0.87 \\
(0.69-1.10)\end{array}$} & \multirow{2}{*}{0.2468} \\
\hline $\mathrm{ARB} / \mathrm{CCB}$ (2 drugs) & 285 & 466 & & \\
\hline \multicolumn{5}{|l|}{ OUTPATIENTS } \\
\hline \multirow[b]{2}{*}{ Drug class } & \multicolumn{2}{|c|}{ No. } & \multirow[b]{2}{*}{$\begin{array}{l}\text { Odds Ratio } \\
(95 \% \mathrm{CI})\end{array}$} & \multirow[b]{2}{*}{$p$} \\
\hline & $\begin{array}{l}\text { With } \\
\text { KD }\end{array}$ & $\begin{array}{l}\text { Without } \\
\text { KD }\end{array}$ & & \\
\hline \multicolumn{5}{|l|}{ Non-IHDRD } \\
\hline $\mathrm{ARB} /$ diuretics (2 drugs) & 134 & 1305 & \multirow{2}{*}{$\begin{array}{c}1.42 \\
(1.17-1.71)\end{array}$} & \multirow{2}{*}{$<0.05$} \\
\hline $\mathrm{ARB} / \mathrm{CCB}$ (2 drugs) & 875 & 12071 & & \\
\hline \multicolumn{5}{|l|}{ Heart failure } \\
\hline $\mathrm{ARB} /$ diuretics (2 drugs) & 309 & 1078 & \multirow{2}{*}{$\begin{array}{c}1.11 \\
(0.95-1.29)\end{array}$} & \multirow{2}{*}{0.1903} \\
\hline ARB/CCB (2 drugs) & 654 & 2527 & & \\
\hline
\end{tabular}

$\mathrm{ARB}$, angiotensin II receptor blockers; $\mathrm{CCB}$, calcium channel blocker; $\mathrm{CI}$, confidence interval; IHDRD, ischemic heart disease related diseases; $\mathrm{KD}$, kidney disease.

\section{CONCLUSION}

In conclusion, most patients with IHDRD and KD were prescribed combination therapy using ARB, which complies with the JSH2014. However, only a very small proportion of patients with heart failure were prescribed the guideline-suggested combination therapy (including a RA-inhibitor and a diuretic). This study provided a clinically important information and demonstrated the utility of NDB not only for the pharmacovigilance study, but also for the verification of therapeutics guideline.

Acknowledgements The authors thank the Japanese Ministry of Health, Labour and Welfare for providing the National Insurance Claims and Health Checkup Database.

Conflict of Interest The authors declare no conflict of interest. 


\section{REFERENCES}

1) Unni S., White K., Goodman M., Ye X., Mavros P., Bash L. D., Brixner D., Am. J. Hypertens., 28, 814-822 (2015).

2) Bakris G. L., Williams M., Dworkin L., Elliott W. J., Epstein M., Toto R., Tuttle K., Douglas J., Hsueh W., Sowers J., Am. J. Kidney Dis., 36, 646-661 (2000).

3) Wald D. S., Law M., Morris J. K., Bestwick J. P., Wald N. J., Am. J. Med., 122, 290-300 (2009).

4) Mahmud A., Feely J., Hypertension, 49, 272275 (2007).

5) Ogihara T., Matsuzaki M., Matsuoka H., Shimamoto K., Shimada K., Rakugi H., Umemoto S., Kamiya A., Suzuki N., Kumagai H., Ohashi Y., Takishita S., Abe K., Saruta T., Hypertens. Res., 28, 331-338 (2005).

6) Ogihara T., Matsuzaki M., Umemoto S., Rakugi H., Matsuoka H., Shimada K., Higaki J., Ito S., Kamiya A., Suzuki H., Ohashi Y., Shimamoto K., Saruta T., Hypertens. Res., 35, 441-448 (2012).

7) Rakugi H., Ogihara T., Umemoto S., Matsuzaki M., Matsuoka H., Shimada K., Higaki J., Ito S., Kamiya A., Suzuki H., Ohashi Y., Shimamoto K., Saruta T., Hypertens. Res., 36, 947-958 (2013).

8) Ogihara T., Kikuchi K., Matsuoka H., Fujita T., Higaki J., Horiuchi M., Imai Y., Imaizumi T., Ito S., Iwao H., Kario K., Kawano Y., Kim-Mitsuyama S., Kimura G., Matsubara H., Matsuura H., Naruse M., Saito I., Shimada K., Shimamoto K., Suzuki H., Takishita S., Tanahashi N., Tsuchihashi T., Uchiyama M., Ueda S., Ueshima H., Umemura S., Ishimitsu T., Rakugi H., Hypertens. Res., 32, 3-107 (2009).

9) Ministry of Health, Labour and Welfare. "Health and Medical Services.": 〈http:// www.mhlw.go.jp / english / wp / wp-hw6 / dl / 02e.pdf $\rangle$, cited 1 February, 2017.
10) Ministry of Health, Labour and Welfare. "Guideline of providing informations about claims data and specific medical checkup.": 〈http://www.mhlw.go.jp/file.jsp?id = 148075 \&name $=0000014030$.pdf $\rangle$, cited 1 February, 2017.

11) Hagiwara H., Nakano S., Ogawa Y., Tohkin M., J. Clin. Pharm. Ther., 40, 273-278 (2015).

12) Tanaka S., Seto K., Kawakami K., J. Pharm. Health Care Sci., 1, 16 (2015).

13) Conen D., Ridker P. M., Buring J. E., Glynn R. J., BMJ, 335, 432 (2007).

14) Gueyffier F., Boutitie F., Boissel J. P., Pocock S., Coope J., Cutler J., Ekbom T., Fagard R., Friedman L., Perry M., Prineas R., Schron E., Ann. Intern. Med., 126, 761-767 (1997).

15) Sairenchi T., Iso H., Irie F., Fukasawa N., Yamagishi K., Kanashiki M., Saito Y., Ota H., Nose T., Hypertens. Res., 28, 901-909 (2005).

16) Brenner B. M., Cooper M. E., de Zeeuw D., Keane W. F., Mitch W. E., Parving H. H., Remuzzi G., Snapinn S. M., Zhang Z., Shahinfar S., N. Engl. J. Med., 345, 861-869 (2001).

17) Kim-Mitsuyama S., Ogawa H., Matsui K., Jinnouchi T., Jinnouchi H., Arakawa K., Kidney Int., 83, 167-176 (2013).

18) Ogawa H., Kim-Mitsuyama S., Matsui K., Jinnouchi T., Jinnouchi H., Arakawa K., Am. J. Med., 125, 981-990 (2012) .

19) Yamaguchi J., Hagiwara N., Ogawa H., Koyanagi R., Kasanuki H., Takagi A., Mori F., Nagashima M., Yagi M., Am. J. Cardiol., 106, 819-824 (2010).

20) Sciarretta S., Palano F., Tocci G., Baldini R., Volpe M., Arch. Intern. Med., 171, 384-394 (2011).

21) Saruta T., Hayashi K., Ogihara T., Nakao K., Fukui T., Fukiyama K., Hypertens. Res., 32, 505-512 (2009). 